\title{
Ameliorative Effect of Pu-erh Tea on DSS-induced Colitis through Regulation of NF-kB Activation in Mice
}

\author{
Yong-Deok Jeon $^{1, *}$ and Su-Jin Kim ${ }^{2, \dagger ; *}$ \\ ${ }^{1}$ Department of Korean Pharmacy, Woosuk University, Wanju-Gun, Jeollabuk-do 55338, Korea \\ ${ }^{2}$ Department of Biotechnology and Convergence, Daegu Haany University, Gyeongsan 38578, Korea
}

\begin{abstract}
Ulcerative colitis (UC), chronic inflammatory bowel disease, is characterized by severe inflammation in the colon. Tea is one of the most popular beverages consumed worldwide. Pu-erh tea, a unique Chinese tea produced by microbial activities, possesses a broad range of health-promoting effects, including anti-aging, anti-Alzheimer's disease, antioxidation and anti-obesity. However, the inhibitory effect of Pu-erh tea on intestinal inflammation and the underlying mechanism remain unclear. The present study was designed to evaluate the regulatory effect of Pu-erh tea extract (PTE) on dextran sulfate sodium (DSS)-induced colitis clinical signs by analyzing the weight loss and colon length in mice. The inhibitory effects of PTE on inflammatory mediators, such as interleukin (IL)- 6 and tumor necrosis factor (TNF)- $\alpha$, and the activation of nuclear factor- $\mathrm{kB}(\mathrm{NF}-\mathrm{kB})$ were also determined in DSS-treated colitis tissue. We observed that PTE treatment significantly inhibited the DSS-induced clinical symptoms of weight loss, decrease,in colon length, and colon tissue damage in mice. Moreover, PTE attenuated the DSS-induced levels of IL- 6 and TNF- $\alpha$ in colon tissue. We also demonstrated the anti-inflammatory mechanism of PTE by suppressing the activation of NF- $\mathrm{kB}$ in DSS-treated colon tissues. Collectively, the findings provide experimental evidence that PTE may be effective in preventing and treatment of intestinal inflammatory disorders, including UC.
\end{abstract}

Key Words: Pu-erh tea; Ulcerative colitis; Dextran sulfate sodium; Nuclear factor- $\kappa \mathrm{B}$

\section{INTRODUCTION}

Ulcerative colitis (UC), a typical inflammatory disorder of colon, can lead to an increased risk of colorectal cancer, and has shown a rapidly increasing trend in recent years (Yashiro, 2014). Typical symptoms of UC include bloody diarrhea, weight loss, and colonic mucosal ulceration inflammation. Numerous studies have shown that there are multifactorial pathological factors involved in UC, including genetic, immune, infection-related and environmental factors
(Choi et al., 2015). Currently, thiopurines, glucocorticosteroid, 5-aminosalicylic acid and immunosuppressive agents have been widely used in clinical therapy of UC (Salice et al., 2019). Unfortunately, side effect of these drugs restrict their clinical application after life- long use. Therefore, there is a need to develop new and effective alternatives for UC.

Cytokines are important for the regulation of immune function. Inflammatory bowel disease is well-known to be associated with increased levels of inflammatory cytokines (Sands and Kaplan, 2007). Interleukin(IL)-6 and tumor necrosis factor (TNF)- $\alpha$ play crucial roles in the progression

Received: November 15, 2021 / Revised: December 14, 2021 / Accepted: December 21, 2021

* Professor.

${ }^{\dagger}$ Corresponding author: Su-Jin Kim. Department of Biotechnology and Convergence, Daegu Haany University, Gyeongsan 38578 , Korea. Tel: +82-53-819-1389, Fax:+82-53-819-1389, e-mail: ksj1009@dhu.ac.kr

(C) The Korean Society for Biomedical Laboratory Sciences. All rights reserved.

(C) This is an Open Access article distributed under the terms of the Creative Commons Attribution Non-Commercial License (http://creativecommons.org/licenses/by-nc/3.0/) which permits unrestricted non-commercial use, distribution, and reproduction in any medium, provided the original work is properly cited. 
of colitis (Mueller, 2002). Studies have shown that blood and colonic tissues from patients with UC exhibit increased IL-6 and TNF- $\alpha$ expression (Park et al., 2017). From this, therapeutic agent that reduces the levels of IL- 6 and TNF- $\alpha$ may effectively ameliorate intestinal inflammation.

Nuclear factor- $\kappa \mathrm{B}(\mathrm{NF}-\kappa \mathrm{B})$ is a kind of transcription factor, which is participated in inflammatory process. Increased $\mathrm{NF}-\kappa \mathrm{B}$ activation has been reported to increase the development of UC due to the induction of cytokines expression (Atreya et al., 2008). Recent studies have demonstrated that intestinal epithelial cells of UC patients exhibit increase of NF- $\kappa$ B activation (Skupsky et al., 2020). Consequently, use of agents that effectively suppress NF- $\mathrm{BB}$ has become an attractive strategy for UC managing.

Tea is one of the most popular beverages worldwide. $\mathrm{Pu}-$ erh tea, one of popular Chinese teas, has a broad range of health-improving properties, including prevention of neurodegenerative disorders, type II diabetes and inhibition of tumor cell progression (Zhao et al., 2005; Du et al., 2012; Liu et al., 2018). However, there has been no information for the mechanism of Pu-erh tea underlying the efficacy of colitis. Therefore, we investigated whether Pu-erh tea can regulate acute colitis symptoms and evaluated its protective mechanim using a dextran sulfate sodium (DSS)-induced experimental colitis models.

The aim of present research was designed to evaluate the regulatory effect of Puer tea extract (PTE) on the clinical features of DSS-induced colitis by measuring body weight loss and colonshortening in mice. The ameliorative effects of PTE on expression of IL-6, TNF- $\alpha$ and activation of $\mathrm{NF}-\kappa \mathrm{B}$ in DSS-treated colon tissue were also determined.

\section{MATERIALS AND METHODS}

\section{Animals and Reagents}

BALB/c mice (male, 6-weeks-old) were purchased from Hyochang Science (Daegu, Korea). The mice were maintained in a SPF environment for at least one week to allow them to adapt the environmental changes. Animals were kept under a 12-h light/dark cycle at room temperature (24 $\left.\pm 2{ }^{\circ} \mathrm{C}\right)$ and humidity $(56 \pm 10 \%)$. DSS was procured from MP Biomedical (Solon, OH, USA). Specific antibody against
NF- $\kappa$ B was purchased from Santa Cruz Biotechnology (Santa Cruz, CA). Nuclear extraction reagent kit and enhanced chemiluminesence (ECL) were procured from Pierce Thermo Scientific (Rockford, IL, USA). The ELISA assay kits for IL-6 and TNF- $\alpha$ were obtained from BD Biosciences (San Diego, CA, USA). 5-aminosalicylic acid (ASA), eosin and hematoxylin and other reagents were purchased from Sigma-Aldrich (St. Louis, MO, USA).

\section{Preparation of PTE}

The dried of Puer tea (200 g) was pulverized into a fine powder and decocted in $1 \mathrm{~L}$ of a distilled water for $3 \mathrm{~h}$, and then concentrated under vacuum. The extract was filtered, freeze drying (Ilshinbiobase, ILSHINBIOBASE-FD, Korea) prior to being maintained at $4{ }^{\circ} \mathrm{C}$ (yield, 15.8\%). The samples were dissolved in PBS and filtered through a $0.22 \mu \mathrm{m}$ syringe filter (GVS ABLUO, Fisher scientific, USA).

\section{Experimental Colitis model construction and treatment}

Colitis in mice was induced by administering 3\% (w/v) DSS drinking water ad libitum for 14 days. The mice were inspected daily for body weight loss, stool consistency and gross bleeding. Mice were divided into 4 groups $(n=6 /$ group): vehicle, DSS, DSS plus PTE (100 mg/kg) and DSS plus ASA (100 mg/kg). PTE and ASA were orally administered during DSS treatment once a day and after which the mice were sacrificed.

\section{Disease activity index (DAI)}

Intestinal disease activity was assessed based on weight loss, the presence of diarrhea accompanied by blood and mucus, and colonic shortening (Hendrickson et al., 2002). The colitis seriousness was measured by DAI assessment, a scoring system which includes three parts as described by Wirtz et al. (2017): body weight loss (0 4), degree of intestinal bleeding $(0 \sim 4)$ and stool consistency $(0 \sim 4)$. The DAI values were determined by three investigators blinded to the protocol.

\section{Histological Evaluation}

The colon tissue was fixed in neutral buffered formalin $(10 \%)$, dehydrated in grade ethanol, and embedded in par- 


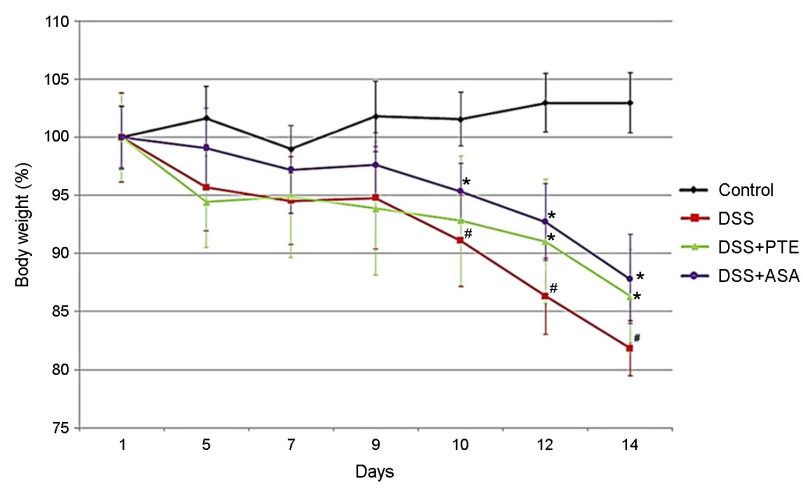

Fig. 1. Improving effect of PTE on DSS-induced the body weight loss in mice. Experimental colitis was induced by administering a $3 \%$ DSS in the drinking water for 14 days. PTE $(100 \mathrm{mg} / \mathrm{kg})$ and ASA (100 mg/kg, the reference drug) were orally administered. Body weight was determined at the same time on the experimental days. Data were represented in the mean \pm S.D. $(n=6)$ and results were analyzed by Tukey post hoc test $\left({ }^{\#} P<0.05\right.$ versus control, ${ }^{*} P$ $<0.05$ versus DSS alone).

affin. Tissue sections were cut at $4 \mathrm{~m}$ on a rotary microtome, mounted on glass slides and dried. Sections were stained with $\mathrm{H} \& \mathrm{E}$ using standard instructions.

\section{Cytokine assay}

Colon tissues were excised, homogenized and extraction by protein extraction solution (Intron Biotechnology, Seoul, Korea). Following protein quantification using bicinchoninic acid protein assay reagent, the levels of IL- 6 and TNF- $\alpha$ of colon tissue were determined using a specific ELISA kit according to the manufacturer's protocols.

\section{Western blot analysis}

Nuclear lysates were prepared by nuclear extraction reagent kit (Pierce Thermo Scientific, Rockford, IL, USA). After protein quantification, the sample was mixed with $2 \mathrm{x}$ sample buffer, separated by gel electrophoresis and transferred to membranes. Membrane was blocked with skimmed milk (5\%) and subsequently reacted with NF- $\mathrm{kB}$ p65 primary Abs. After washing, the membrane was incubated with secondary Abs for $1 \mathrm{~h}$. After washing, protein bands were visualized using an enhanced chemiluminescence detection system (Thermo Fisher Scientific Inc. NJ, USA).

\section{Statistical analysis}

Results are represented as the mean \pm S.D. The statistical analyses were performed using an independent $t$-test and ANOVA with a Tukey post hoc test. $P<0.05$ was considered significant.

\section{RESULTS}

\section{Improving effect of PTE on DSS-induced weight loss}

The DSS-caused colitis model resemble human symptoms of UC (Eichele et al., 2017). The DSS-induced clinical features (body weight loss, bloody diarrhea and colon shortening) were confirmed in this model. First, regulatory effect of PTE on the weight loss in DSS-caused colitis was determined. We observed that PTE group showed significant prevention of body weight loss induced by DSS (Fig. 1). Additionally, any other adverse except colitis features did not observed in PTE group. ASA has been used as a reference drug.

\section{Improving y effect of PTE on DSS-induced colon short- ening}

Shortening of colon length is a morphological indicator to determine degree of colitis symptoms (Nunes et al., 2018). We evaluated improving effect of PTE on the colon length shortening in DSS-induced colitis.

As shown in Fig. 2A, the colon length in DSS-treated group was shorter than those of the control mice. However, PTE treatment alleviated the effect of DSS on colon shortening. Relative colon length has been represented in Fig. 2B.

\section{Improvingeffect of PTE on DSS-induced disease activity index (DAI) and colonic injury}

DAI is a parameter to determine degree of colitis symptoms (Wirtz et al., 2017). In order to evaluate the therapeutic effects of PET on DSS induced colitis, the DAI were recorded. Our results indicated that DAI score significantly increased in DSS-treated group, whereas it was markedly attenuated in PTE group (Fig. 3A). Additionally, histological analysis of the colon showed that DSS-induced colonic injuries, such as epithelial crypt and lamina propria damage, 
A

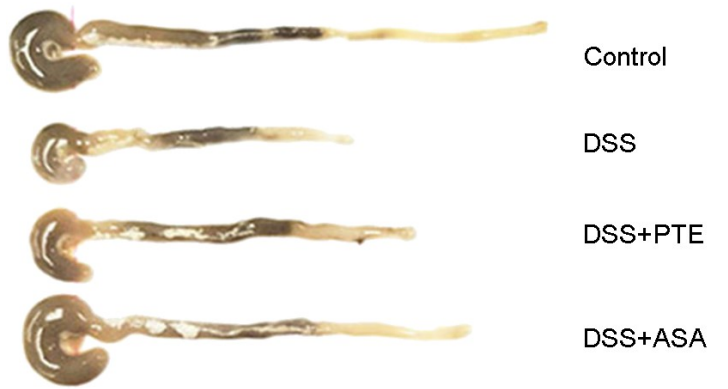

B

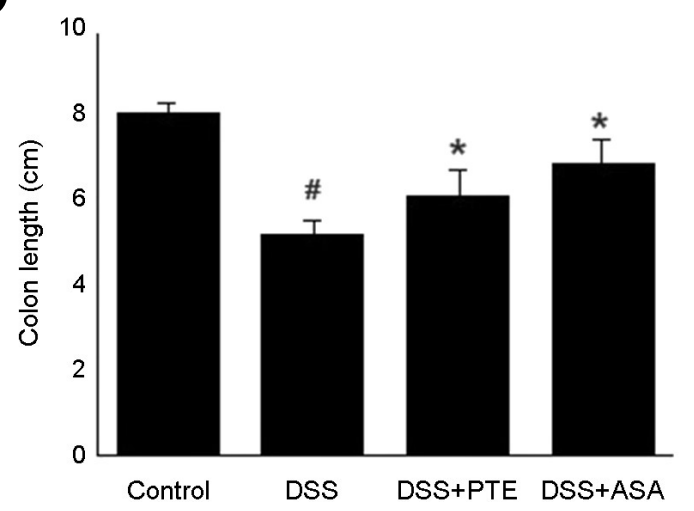

Fig. 2. Improving effect of PTE on DSS-induced the colon length shortening in mice. (A) The colons were removed at day 14 after DSS treatment, and the lengths of colon were measured. (B) Relative colon lengths were represented. Data were represented in the mean \pm S.D. $(n=6)$ and results were analyzed by Tukey post hoc test ( ${ }^{\sharp} P<0.05$ versus control, ${ }^{*} P<0.05$ versus DSS alone).

was significantly alleviated after treatment with PTE (Fig. 3B). As shown in Fig. 3C, the thickness of colon muscle layer were represented.

\section{Regulatory effect of PTE on DSS-induced inflammatory cytokine levels in colon tissue}

As an increase in inflammatory cytokine levels is associated with the physiological processes of UC (Ogata and Hibi, 2003), we investigated the effect of PTE on IL-6 and TNF- $\alpha$ expression in DSS-induced colon tissue. Colon tissue was excised and homogenized after the end of experiment. As shown in Fig. 4, DSS induced IL-6 and TNF- $\alpha$ expression compared to the controls. However, enhanced IL- 6 and TNF- $\alpha$ expression cause by DSS was markedly inhibited by PTE and ASA treatment in colon tissue.
(A)

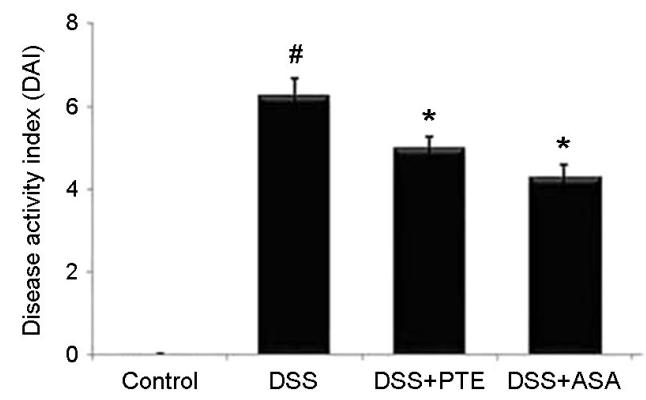

B
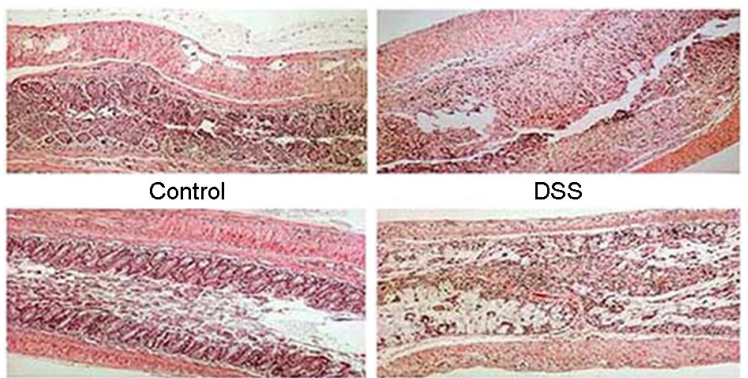

DSS+PTE
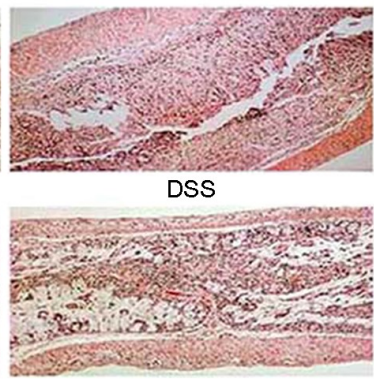

DSS+ASA

C

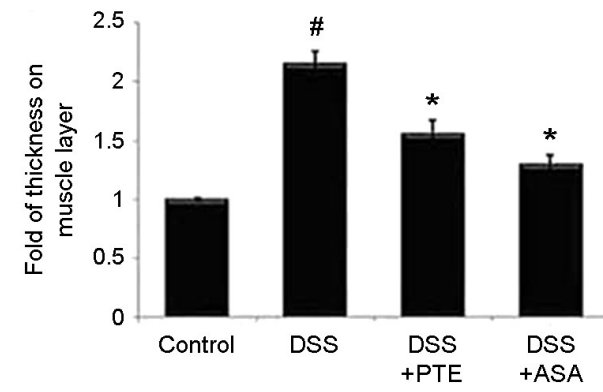

Fig. 3. Improving effect PTE on DSS-induced the DAI increase and colonic injury in mice. (A) DAI was calculated as described in Materials and Methods. (B) H\&E staining was performed to evaluate the effect of PTE. (C) The relative thickness of muscle layer were represented. Data were shown in the mean \pm S.D. $(\mathrm{n}=$ 6 ) and results were analyzed by Tukey post hoc test $\left({ }^{\sharp} P<0.05\right.$ versus control, ${ }^{*} P<0.05$ versus DSS alone).

\section{Regulatory effect of PTE on DSS-induced NF-кB acti- vation in colon tissue}

To determine the molecular mechanism of PTE involvement in inflammatory progress of $\mathrm{UC}$, we evaluated that PTE regulate the DSS-induced NF- $\kappa B$ activation. NF- $\kappa B$ activation was remarkably enhanced in colon tissues of DSS-treated mice, and PTE administration attenuated the DSS-induced NF- $\kappa$ B activation (Fig. 5A). Relative levels of NF- $\kappa B$ were shown using an image analyzer (Fig. 5B). 

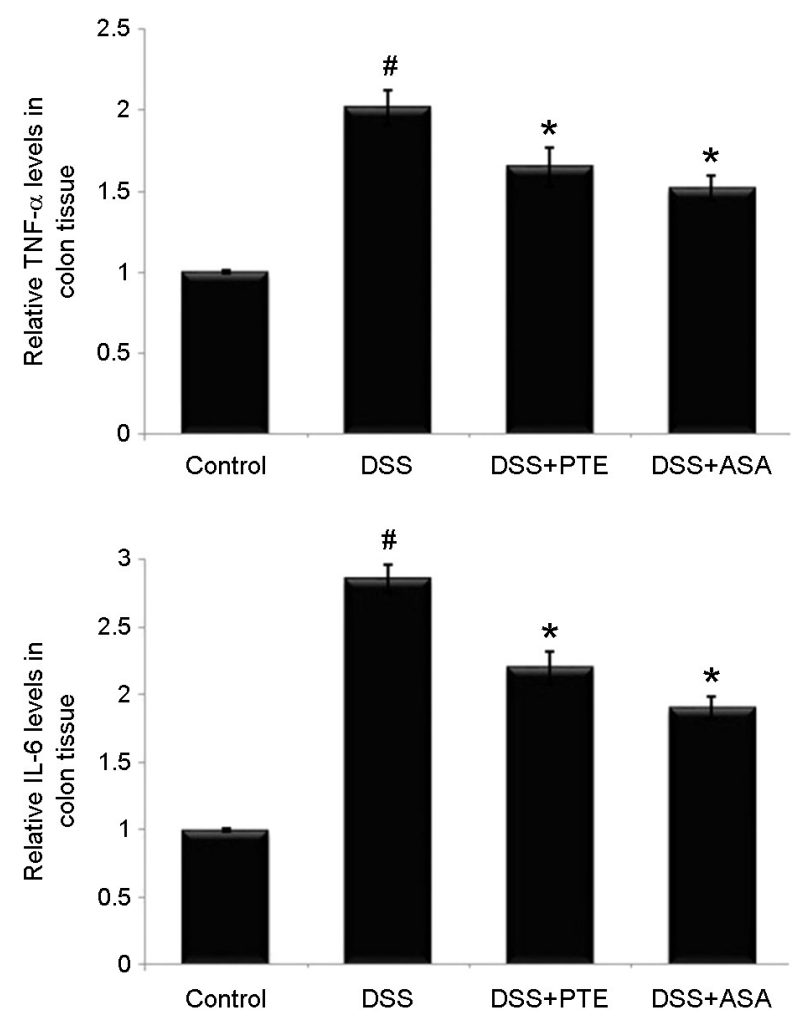

Fig. 4. Regulatory effect of PTE on the inflammatory cytokines levels in DSS-induced colitis tissue. At the end of the experiment, the colon tissues were excised and homogenized. Following protein quantification, the levels of IL- 6 and TNF- $\alpha$ in colonic tissue were assayed using ELISA assay kits (Data shown are expressed as the means \pm S.D. $\left({ }^{\#} P<0.05\right.$ versus control, ${ }^{*} P<0.05$ versus DSS alone).

\section{DISCUSSION}

$\mathrm{Pu}$-erh tea is not only a popular tea, but also used in traditional Chinese medicine, which has many health-improving properties such as anti-oxidant, anti-obesity and anti-cancer and so on. However, information on the precise mechanism of Pu-erh tea on intestinal inflammation including UC remains limited. The finding of this study demonstrated that PTE suppress colitis clinical signs, colonic injury and inflammatory response induced by DSS, suggesting a mechanism by which PTE ameliorates intestinal inflammation in mice.

$\mathrm{UC}$ is a inflammatory intestinal disorder and can lead to an increased risk of colorectal cancer. The features of UC are characterized by colonic mucosal ulceration, bloody diarrhea, and abdominal pain. Many other complicated factors, including genetic, immune, infection-related and environ-
A

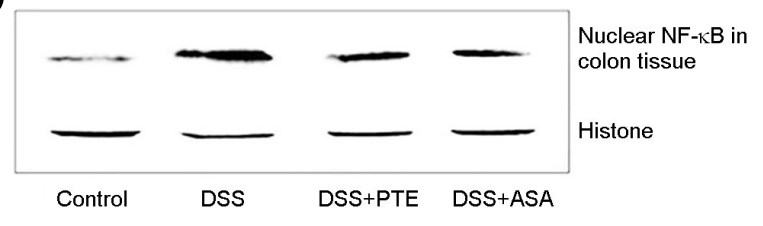

B

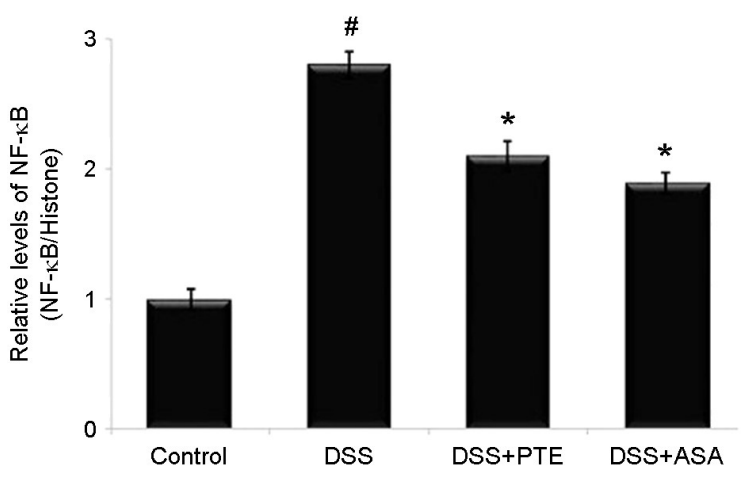

Fig. 5. Regulatory effect of PTE on the NF- $\kappa B$ activation in in DSS-treated colon tissue. The colon tissues were excised and homogenized. Following protein quantification, nuclear extracts in colonic tissue were prepared by nuclear extraction reagent kit. (A) The levels of NF-кB p65 were assayed by Western blot analysis. (B) The relative ratio of NF- $\mathrm{kB}$ p65 was calculated using an image analyzer. Data shown are expressed as the means \pm S.D. $\left({ }^{\sharp} P<\right.$ 0.05 versus control, ${ }^{*} P<0.05$ versus DSS alone).

mental factors, are involved in the pathogenesis of UC (Danese et al., 2004). Therapeutic agents for UC treatment include glucocorticosteroids, 5-ASA, sulfasalazine, immunosuppressants (Domenech, 2006). However, these have serious side effects for long-term use. Therefore, new and effective alternative agents for UC need to be developed. In this study, we determined the improving effect of PTE in DSS-induced colitis. The DSS-induced clinical signs of UC are body weight change, colon shortening and bloody diarrhea (Ardizzone and Bianchi, 2005). We confirmed that mice treated with PTE suppressed body weight loss and colon shortening induced by DSS. DAI value, which were scored using major clinical features (body weight changes, diarrhea, and rectal bleeding extent), was markedly higher than that of the control. However, DAI increases caused by DSS were markedly inhibited in groups administered PTE. Additionally, histological analysis of the colon showed that treatment with PTE improved DSS-induced colonic injury, such as epithelial crypt and lamina damage. These results demonstrated 
that PTE exerts anti-colitis activity by regulation the clinical symptoms of colitis and colonic injury caused by DSS in mice. Recent reports have shown that inflammatory cytokine is associated with the pathogenesis of intestinal inflammation, including UC (Mueller, 2002). IL-6 and TNF- $\alpha$ levels have been reported to be elevated in patients with UC and play an integral role in UC pathogenesis (Sands and Kaplan, 2007). Another previous study found that IL- 6 and TNF- $\alpha$ are key contributors to UC carcinogenesis (Mudter and Neurath, 2007). Thus, we determined the ameliorative effect of PTE the IL- 6 and TNF- $\alpha$ expression levels in DSS-induced colon tissue. Our results show that the PTE inhibited the DSSinduced increase of IL-6, TNF- $\alpha$ expression in colon tissue. Thus, we suggest that anti-inflammatory activity of PTE might be associated with the regulation of inflammatory cytokines, including IL-6, TNF- $\alpha$ in DSS-induced colon tissue.

Numerous reports have shown that NF- $\kappa \mathrm{B}$ activation is involved in pathogenesis of UC (Tak and Firestein, 2001). Increase of inflammatory cytokines is dependent on activation of NF- $\mathrm{kB}$ during intestinal inflammation (Skupsky et al., 2020). NF-kB has been reported to be highly expressed in intestinal mucosal epithelium, epithelial cells and crypt of patients with UC (Chung et al., 2014). Additionally, several studies have shown that NF- $\kappa$ B plays an integral role in the disruption of epithelial barrier function (Atreya et al., 2008). Therefore, inhibitions of NF- $\kappa B$ activation have been demonstrated as a novel strategy for UC. Our results revealed that DSS-induced NF- $\kappa \mathrm{B}$ activation was remarkably inhibited by PTE treatment in colitis tissue. These results indicated that PTE attenuates intestinal inflammation by blocking NF$\kappa \mathrm{B}$ activation in colitis. Although we confirmed the role of PTE in the attenuation of NF- $\kappa \mathrm{B}$ activation in colitis, the regulatory mechanism of PTE on other pathways were not elucidated. Therefore, further studies are required to determine the exact mechanism of PTE in regulation of intestinal inflammatory response.

Collectively, our results indicate that PTE possess anticolitis activity by relieving of the clinical symptoms and colonic damage against DSS-induced colitis. Moreover, we demonstrate that anti-colitis effect of PTE can attributed to the suppression of NF- $\mathrm{kB}$ activation and subsequent down- regulation of inflammatory cytokines expression levels in colon tissue. These results provide experimental evidence that PTE might be an efficacious and promising therapeutic candidate for treatment of intestinal inflammatory disorders, including UC.

\section{ACKNOWLEDGEMENT}

This research was supported by "Kuaihuo Puercha" Institute.

\section{CONFLICT OF INTEREST}

No potential conflict of interest relevant to this article was reported.

\section{REFERENCES}

Atreya I, Atreya R, Neurath MF. NF-kappaB in inflammatory bowel disease Journal of Internal Medicine. 2008. 263: 591596.

Ardizzone S, Bianchi PG. Biologic therapy for inflammatory bowel disease. Drugs. 2005. 65: 2253-2286.

Choi Y, Koh SJ, Lee HS, Kim JW, Kim BG, Lee KL, Kim JS. Roxithromycin inhibits nuclear factor kappaB signaling and endoplasmic reticulum stress in intestinal epithelial cells and ameliorates experimental colitis in mice. Experimental Biology and Medicine. 2015. 240: 1664-1671.

Chung CY, Park YL, Kim N, Oh HH, Myung DS, Kim JS, Cho SB, Lee WS, Kim HS, Ahn BW, Joo YE. Rice prolamin extract ameliorates acute murine colitis by inhibiting nuclear factor-kappa B and modulating intestinal apoptosis and cell proliferation. Clinical and Experimental Immunology. 2014. 178: 537-547.

Danese S, Sans M, Fiocchi C. Inflammatory bowel disease; the role of environmental factors. Autoimmunity Reviews. 2004. 3: $394-400$

Domenech E. Inflammatory bowel disease: current therapeutic options. Digestion. 2006. 73: 67-76.

Du WH, Peng SM, Liu ZH, Shi L, Tan LF, Zou XQ. Hypoglycemic effect of the water extract of Pu-erh tea. Journal of Agricultural and Food Chemistry. 2012. 60: 10126-10132.

Eichele DD, Kharbanda KK. Dextran sodium sulfate colitis murine model: An indispensable tool for advancing our understanding of inflammatory bowel diseases pathogenesis. World Journal 
of Gastroenterology. 2017. 23: 6016-6029.

Hendrickson BA, Gokhale R, Cho JH. Clinical aspects and pathophysiology of inflammatory bowel disease. Clinical Microbiology Reviews. 2002. 15: 79-94.

Liu Z, Xie HL, Chen L, Huang JH. An Improved Weighted Partial Least Squares Method Coupled with Near Infrared Spectroscopy for Rapid Determination of Multiple Components and Anti-Oxidant Activity of Pu-Erh Tea. Molecules. 2018. 23: 1058.

Mudter J, Neurath MF. Il-6 signaling in inflammatory bowel disease: pathophysiological role and clinical relevance. Inflammatory Bowel Diseases. 2007.13: 1016-1023.

Mueller C. Tumour necrosis factor in mouse models of chronic intestinal inflammation. Immunology. 2002. 105: 1-8.

Nunes NS, Kim S, Sundby M, Chandran P, Burks SR, Paz AH, Frank JA. Temporal clinical, proteomic, histological and cellular immune responses of dextran sulfate sodium-induced acute colitis. World J Gastroenterol. 2018. 24: 4341-4355.

Ogata H, Hibi T. Cytokine and anti-cytokine therapies for inflammatory bowel disease. Current Pharmaceutical Design. 2003. 9: 1107-1113.

Park JH, Peyrin-Biroulet L, Eisenhut M, Shin J. IBD immunopathogenesis: a comprehensive review of inflammatory molecules. Autoimmunity Reviews. 2017. 16: 416-426.

Skupsky J, Sabui S, Hwang M, Nakasaki M, Cahalan MD, Said HM. Biotin supplementation ameliorates murine colitis by preventing NF-kappaB activation. Cell Mol Gastroenterol Hepatol. 2020. 9: 557-567.

Salice M, Rizzello F, Calabrese C, Calandrini L, Gionchetti P. A current overview of corticosteroid use in active ulcerative colitis. Expet Review of Gastroenterology \& Hepatology. 2019. 13: $557-561$.

Sands BE, Kaplan GG. The role of TNFalpha in ulcerative colitis. Journal of Clinical Pharmacology. 2007. 47: 930-941.

Tak PP, Firestein GS. NF-kB: A key role in inflammatory disease. Journal of Clinical Investigation. 2001. 107: 7-11.

Wirtz S, Popp V, Kindermann M, Gerlach K, Weigmann B, Fichtner-Feigl S, Neurath MF. Chemically induced mouse models of acute and chronic intestinal inflammation. Nature Protocols. 2017. 12: 1295-1309.

Yashiro M. Ulcerative colitis-associated colorectal cancer. World. Journal of Gastroenterology. 2014. 20: 16389-16397.

Zhao LF, Zhou HJ, An WJ. Study on health promoting effect of Yunnan Pu-erh tea. Shipin Yanjiu Yu Kaifa. 2005. 26: 114-118.

https://doi.org/10.15616/BSL.2021.27.4.248

Cite this article as: Jeon YD, Kim SJ. Ameliorative Effect of Pu-erh Tea on DSS-induced Colitis through Regulation of NF- $\mathrm{BB}$ Activation in Mice. Biomedical Science Letters. 2021. 27: 248-254. 